\title{
Temperatura e umidade do solo em função do uso de técnica nucleadora no Semiárido
}

\author{
Islanny Alvino Leite ${ }^{1 *}$, Rennan Salviano Terto ${ }^{1}$, Andreza Ferreira Guedes ${ }^{1}$, Matthaus Klisnmann da Costa Silva ${ }^{1}$, \\ Francisco de Assis Pereira Leonardo ${ }^{1}$, Jacob Silva Souto ${ }^{1}$
}

\begin{abstract}
RESUMO: Este estudo objetivou avaliar a temperatura e o conteúdo de água do solo em diferentes profundidades em função do uso da técnica nucleadora de transposição de galhadas no Núcleo de Desertificação do Seridó, Semiárido da Paraíba. O estudo foi realizado no período de fevereiro de 2015 a fevereiro de 2016, na Fazenda Cachoeira de São Porfírio, município de Várzea, Paraíba. Utilizou-se delineamento experimental em blocos ao acaso, com cinco tratamentos representados por diferentes distâncias entre as galhadas, em quatro repetições. Em cada parcela, foram instaladas quatro galhadas (abrigos artificiais). Foi analisado mensalmente o conteúdo de água no solo e a temperatura do solo no interior e fora das galhadas nas profundidades 0,$0 ; 7,5$ e $15 \mathrm{~cm}$. Aplicou-se a análise de variância e o teste de Tukey a 5\% de significância. A análise da temperatura do solo revelou interação significativa entre as três profundidades e os doze meses de estudo $(\mathrm{p}<0,05)$. O regime térmico do solo foi influenciado pela utilização das galhadas, pois as menores amplitudes térmicas ocorreram no interior das galhadas em todos os meses estudados. Conclui-se que, nas condições de presença de galhadas, a temperatura do solo apresentou menores amplitudes em relação ao solo descoberto.
\end{abstract}

Palavras-chave: abrigos artificiais, desertificação, amplitude térmica, conteúdo de água do solo.

\section{Soil temperature and humidity due to the use of nucleating technique in Semi-arid}

\begin{abstract}
The objective of this study was to evaluate soil temperature at different depths as a function of the use of the nucleating technique of transposition of branches in the Desertification Nucleus of Serido, Paraíba. The study was carried out from February 2015 to February 2016 at Fazenda Cachoeira de São Porfírio, municipality of Várzea-PB. A randomized complete block design was used, with five treatments represented by different distances between the branches in four replications. In each plot, four branches (artificial shelters) were installed. The soil water content and soil temperature were analyzed monthly in and out of the branches at depths of 0.0; 7.5 and $15 \mathrm{~cm}$. The analysis of variance and the Tukey test were applied at 5\% significance. Soil temperature analysis revealed significant interaction between the three depths and the twelve months of study $(p<0.05)$. The thermal regime of the soil was influenced by the use of branches, because the smaller thermal amplitudes occurred inside the branches in all months studied. It was concluded that, under the conditions of presence of branches, the soil temperature presented smaller amplitudes in relation to the uncovered soil.
\end{abstract}

Keywords: artificial shelters, desertification, thermal amplitude, soil water content.

\section{INTRODUÇÃO}

A desertificação é um processo de degradação das terras que ocorre nas regiões áridas, semiáridas e subúmidas secas no mundo. No Nordeste do Brasil, as condições climáticas e sobretudo a acentuada evaporação, baixos índices pluviométricos e o uso da terra em meio à falta de políticas públicas agrárias efetivas concorrem para aumentar o risco de desertificação na região (FIGUEIREDO, 2013). O Núcleo de Desertificação do Seridó apresenta uma área de $2.987 \mathrm{~km}^{2}$ e abrange alguns municípios do Rio Grande do Norte e da Paraíba (Santa Luzia, Várzea) (PEREZ-MARIN et al., 2012).

Reis et al. (2010) discutem o conceito de nucleação em uma perspectiva de restauração da conectividade de paisagens fragmentadas. Os autores consideram a nucleação como um processo envolvendo qualquer elemento, biológico ou abiótico, capaz de propiciar potencialidades para formar, dentro de comunidades em restauração, novas populações através da facilitação e criação de novos nichos de regeneração/colonização e gerando novas situações de conectividade na paisagem.

A técnica de transposição de galhada consiste no acúmulo de galhos, troncos e resíduos florestais, formando núcleos, atuando como refúgios artificiais para a fauna, por apresentar um clima mais favorável. Ações nucleadoras como o uso da técnica de transposição de galhadas representam um avanço em modelos de restauração, expressando o forte caráter ético com a conservação e manejo das paisagens (REIS et al., 2014).

Ramana Rao et al. (2005) ressaltam que são importantes as observações regulares da temperatura do solo em diferentes profundidades, pois possibilitam identificar seu comportamento térmico e o conhecimento de importantes propriedades que

\footnotetext{
Recebido em 05/07/2019, Aceito para publicação em 15/10/2019

${ }^{1}$ Universidade Federal de Campina Grande

*e-mail: islanny_alvino@hotmail.com
} 
podem ser utilizadas em outros trabalhos, tais como difusividade e condutividade térmica.

Diante do exposto, o presente trabalho objetivou avaliar a temperatura do solo em diferentes profundidades em condições de solo descoberto e na presença da técnica nucleadora de transposição de galhadas na Fazenda Cachoeira de São Porfírio, município de Várzea-PB.

\section{MATERIAL E MÉTODOS}

O estudo foi desenvolvido na Fazenda Cachoeira de São Porfírio, que possui uma área de 58 hectares,situada no município de Várzea-PB (06² 48'32.01'’S; 36 57'17.04'W), microrregião do Seridó Ocidental, Mesorregião da Borborema, com altitude de $271 \mathrm{~m}$, no Núcleo de Desertificação do Seridó.

A área de estudo apresenta solos de origem cristalina. São rasos, pedregosos e apresentam elevada suscetibilidade à erosão, predominando NeossolosFlúvicos distróficos. Segundo a classificação de Köppen (1996) o clima da região se enquadra no tipo BSh (semiárido) quente e seco, com médias anuais térmicas superiores a $25^{\circ} \mathrm{C}$ e pluviosidade média anual inferior a $800 \mathrm{~mm}^{2} \mathrm{ano}^{-1}$, com chuvas irregulares.

A pesquisa foi desenvolvida no período de fevereiro de 2015 a fevereiro de 2016, em área demonstrativa de 4,0 hectares, isolada por meio de cerca de arame farpado. $O$ experimento foi conduzido utilizando-se delineamento em blocos ao acaso, com cinco tratamentos em quatro repetições. Cada parcela experimental foi composta por quatro galhadas (abrigos artificiais) com dimensões de 0,7 $\mathrm{m} \times 0,7 \mathrm{~m} \times 0,7 \mathrm{~m}$.

Os tratamentos utilizados foram representados por diferentes distâncias entre as galhadas (I: 5,0 m x 5,0 m; II: $10,0 \mathrm{~m}$ x $10,0 \mathrm{~m}$; III: $15,0 \mathrm{~m}$ x $15,0 \mathrm{~m}$; IV: $20,0 \mathrm{~m} \times 20,0 \mathrm{~m}$; $\mathrm{V}$ : controle) com quatro repetições. $\mathrm{O}$ tratamento controle foi distribuído em área de preservação da caatinga, contígua a área experimental, obedecendo a espaçamentos aleatórios dentro da área. As galhadas foram formadas por galhos e troncos coletados em áreas adjacentes, prevalecendo galhos da espécie CrotonblanchetianusBaill. (Marmeleiro) por apresentar muitos indivíduos no local. As galhadas foram demarcadas por quatro piquetes nas extremidades e preenchidas com galhos até a altura de $0,7 \mathrm{~m}$ (Figura 1).

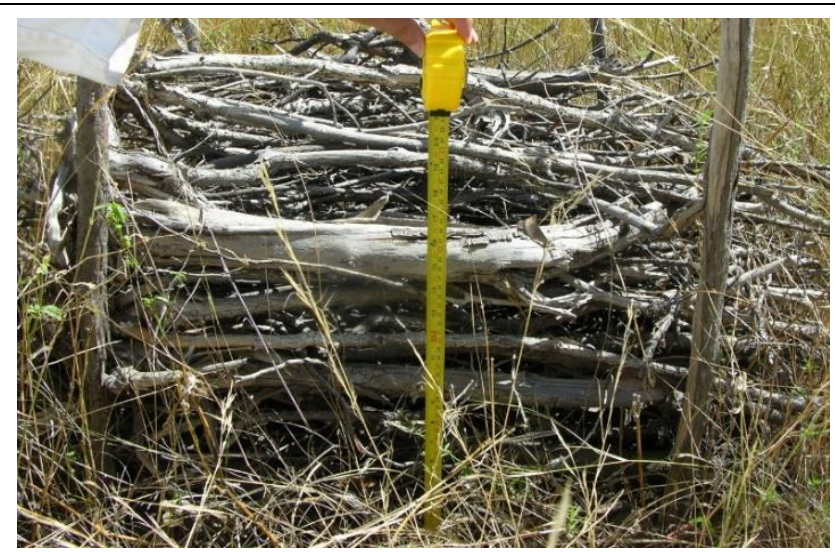

Figura 1 - Galhadas instaladas no núcleo experimental. Fonte-Autoria própria (2015).

Verificou-se a temperatura do solo mensalmente nos cinco tratamentos, no interior e fora das galhadas, nas profundidades 0,$0 ; 7,5$ e $15,0 \mathrm{~cm}$, utilizando-se termômetro digital portátil modelo DIGITAL - TermometerFrenchCooking.

As análises do conteúdo de água do solo foram realizadas mensalmente. Após as coletas, as amostras foram pesadas em balança analítica para obter o peso de massa de solo úmido $(\mathrm{g})$ e, em seguida, colocadas em estufa a uma temperatura de $105^{\circ} \mathrm{C}$, por 24 horas, para obter o peso da massa seca das amostras $(\mathrm{g})$. O conteúdo de água foi calculado através da seguinte fórmula:

Em que:

$$
U=\frac{M u-M s}{M s} \times 100
$$

U - conteúdo de água (\%);Mu - massa de solo úmido (g); Ms - massa de solo seco (g).

Os dados da precipitação pluviométrica utilizados neste estudo foram obtidos por meio da instalação de pluviômetro na sede da propriedade, a uma distância de 1,0 kmda área experimental. Os dados foram submetidos à análise de variância para avaliação dos efeitos dos tratamentos. As médias foram comparadas pelo teste de Tukey a $5 \%$ de significância. As análises estatísticas foram realizadas utilizando o Software - SAS/STAT 9.3 (2011).

\section{RESULTADOS E DISCUSSÕES}

A precipitação durante o período de coleta de dados totalizou $714,3 \mathrm{~mm}$, caracterizando uma estação seca (julho a dezembro/2015) e outra chuvosa (março a junho/2015 e janeiro/2016) (Figura 2). 


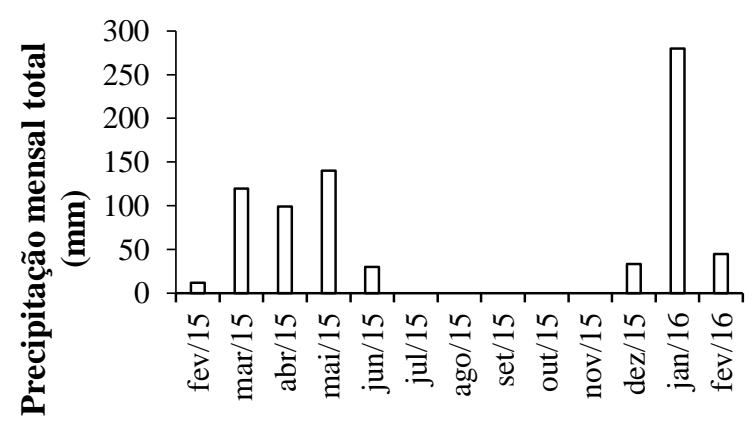

Meses

Figura 2-Precipitação pluviométrica mensal total (mm) ocorridas na Fazenda Cachoeira de São Porfírio, município de Várzea - PB, de fevereiro de 2015 a fevereiro de 2016.

Fonte - Dados da pesquisa (2016).

A tabela 1 apresenta a análise de variância da temperatura do solo analisada ao nível de $5 \%$ de significância $(\mathrm{p}<0,05)$ em três profundidades $(0,0$; 7,5 e $15,0 \mathrm{~cm}$ ) nos doze meses de estudo.

Tabela 1.Temperaturas médias $\left({ }^{\circ} \mathrm{C}\right)$ coletadas em diferentes profundidades no Núcleo de Desertificação do Seridó, Semiárido da Paraíba.

\begin{tabular}{cccc}
\hline & \multicolumn{3}{c}{ Profundidade $(\mathrm{cm})$} \\
\cline { 2 - 4 } Meses & 0,0 & 7,5 & 15,0 \\
\hline Mar/15 & $28,1 \mathrm{Aef}$ & $27,7 \mathrm{Ae}$ & $27,9 \mathrm{Af}$ \\
$\mathrm{Abr} / 15$ & $32,4 \mathrm{Ac}$ & $31,7 \mathrm{Ac}$ & $31,3 \mathrm{Ade}$ \\
$\mathrm{Mai} / 15$ & $29,1 \mathrm{Ae}$ & $28,5 \mathrm{Ae}$ & $28,6 \mathrm{Af}$ \\
$\mathrm{Jun} / 15$ & $29,4 \mathrm{Ade}$ & $30,1 \mathrm{Ad}$ & $30,3 \mathrm{Ae}$ \\
$\mathrm{Jul} / 15$ & $32,4 \mathrm{Ac}$ & $31,7 \mathrm{Ac}$ & $31,3 \mathrm{Ade}$ \\
$\mathrm{Ago} / 15$ & $32,4 \mathrm{Ac}$ & $31,4 \mathrm{ABcd}$ & $30,9 \mathrm{Be}$ \\
$\mathrm{Set} / 15$ & $33,6 \mathrm{Abc}$ & $32,6 \mathrm{Abc}$ & $32,6 \mathrm{Acd}$ \\
$\mathrm{Out} / 15$ & $35,7 \mathrm{Aa}$ & $34,3 \mathrm{Ba}$ & $34,6 \mathrm{ABab}$ \\
Nov/15 & $35,8 \mathrm{Aa}$ & $34,2 \mathrm{Ba}$ & $34,9 \mathrm{ABa}$ \\
Dez/15 & $34,7 \mathrm{Aab}$ & $34,1 \mathrm{Aab}$ & $35,1 \mathrm{Aa}$ \\
Jan/16 & $30,8 \mathrm{Bd}$ & $32,3 \mathrm{Ac}$ & $33,2 \mathrm{Abc}$ \\
Fev/16 & $27,1 \mathrm{Af}$ & $27,1 \mathrm{Ae}$ & $27,4 \mathrm{Af}$ \\
\hline
\end{tabular}

Médias seguidas de mesma letra maiúscula na linha (profundidade) e minúscula na coluna (meses) não diferem entre si pelo teste de Tukey a 5\% de probabilidade.

A análise de variância da temperatura do solo analisada ao nível de $5 \%$ de significância $(\mathrm{p}<0,05)$ revelou interação significativa entre as três profundidades $(0,0 ; 7,5$ e $15,0 \mathrm{~cm})$ e os doze meses de estudo, indicando que as temperaturas do solo variam em função da profundidade e dos meses em estudo.

Esses resultados corroboram o encontrado por Gomes (2015), quando avaliou a temperatura do solo também a 0,0; 7,5 e 15,0 cm de profundidade, às 9h00min, na Fazenda Cachoeira de São Porfírio, em Várzea-PB, concluindo que, à medida que a profundidade aumentava, ocorria uma diminuição na temperatura do solo, demonstrando a relação entre a diminuição da amplitude de variação da temperatura do solo e o aumento da profundidade. Para
Gasparimet al. (2005), a temperatura do solo é um dos fatores mais importantes para a germinação de sementes e desenvolvimento das plantas.

Devido à variação na temperatura ser resultante do fluxo de calor no solo, torna-se um componente necessário ao balanço de energia oriundo da superfície; sendo este, portanto, capaz de justificar o armazenamento e a transferência de calor dentro do solo e, ainda, as trocas entre o solo e a atmosfera (MOURA, QUERINO, 2010).

$\mathrm{Na}$ figura 3 observa-se a análise estatística da temperatura do solo no interior e fora das galhadas $(\mathrm{p}<0,05)$. Observa-se que o regime térmico do solo na área em estudo foi influenciado pela utilização das galhadas. A análise estatística da temperatura do solo demonstrou diferença significativa entre as médias no interior e fora das galhadas $(\mathrm{p}<0,05)$.

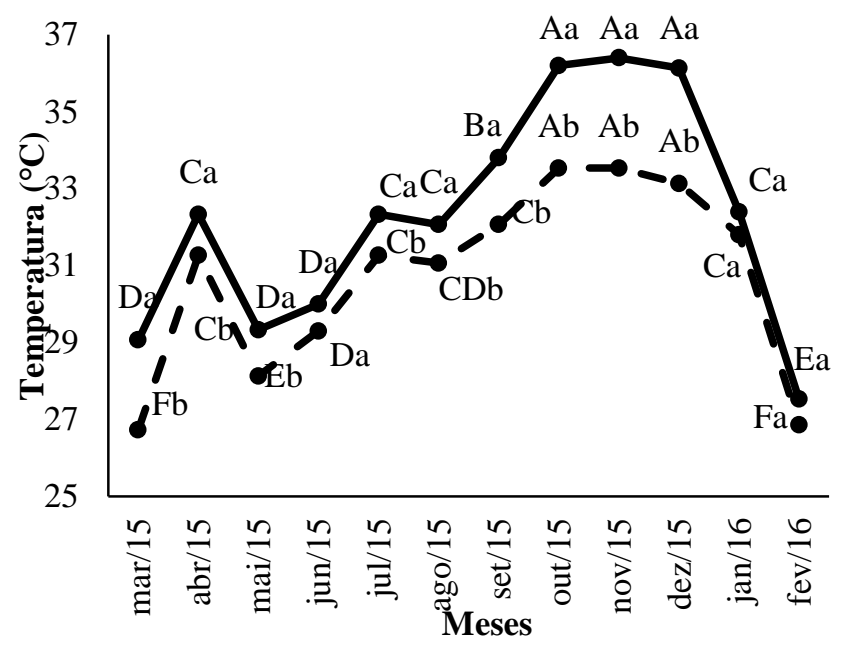

Figura 3 - Temperatura média do solo $\left({ }^{\circ} \mathrm{C}\right)$ no interior (- - -)e fora das galhadas (-) nos meses de estudo no Núcleo de Desertificação do Seridó, semiárido da Paraíba.

Médias seguidas de mesma letra maiúscula (meses) e minúscula (local) não diferem entre si pelo teste de Tukey a $5 \%$ de probabilidade.

Observa-se que as menores amplitudes térmicas ocorreram no interior das galhadas. A superfície do solo, com ou sem cobertura vegetal, exerce importante função sobre sua temperatura, uma vez que a cobertura vegetal é responsável pela troca e armazenamento de energia térmica nos ecossistemas terrestres (CARNEIRO et al., 2013).

A análise estatística dos locais em estudo (dentro e fora da galhada), revela que não houve diferença significativa nos meses de junho/2015, janeiro/2016 e fevereiro/2016. Nos demais meses, a maior temperatura do solo foi observada fora das galhadas, indicando a eficiência das mesmas em manter a temperatura do solo baixa, proporcionando abrigos para animais dispersores de sementes da fauna local, vindo de locais próximos (mata preservada), como enfatizam os autores Silveira et al. (2015), que, quanto mais próxima uma área a ser recuperada 
estiver de uma área com vegetação nativa, mais rápida e intensa será a chegada de sementes trazidas pelos agentes dispersores.

De acordo com Belan et al. (2013), os resíduos vegetais depositados na superfície protegem o solo contra o aquecimento excessivo e a perda de água.

Constata-se que, nos meses de setembro a dezembro/2015 (Figura 3), as temperaturas apresentaram uma redução de aproximadamente $4^{\circ} \mathrm{C}$ com a utilização das galhadas, por ser o período mais quente do ano e apresentar pouca distribuição de chuvas, revelando a importância das galhadas, que se tornam eficazes cobrindo e protegendo o solo das altas temperaturas. No estudo realizado por Belan et al. (2013), a temperatura no perfil do solo variou em função da profundidade e da condição de cobertura. Ao estudarem sob duas condições de cobertura, os autores perceberam que, na condição de ausência de cobertura, a temperatura do solo apresentou maiores amplitudes ao longo do dia em relação ao solo coberto, e tais amplitudes variam entre as diferentes profundidades analisadas.

Gomes (2015), estudando temperaturas do solo no Núcleo de Desertificação do Seridó, em Várzea$\mathrm{PB}$, observou que a amplitude térmica na superfície do solo foi alta, atingindo $9,75{ }^{\circ} \mathrm{C}$, e que essa variação pode ter ocorrido em virtude da pouca cobertura do solo no período de coleta. Ressaltou, ainda, que a cobertura do solo nas áreas em processo de desertificação no Semiárido brasileiro é uma técnica imprescindível, pois protege o solo da incidência direta dos raios solares e evita a perda de água por evaporação.

O conteúdo de água no solo versus a temperatura do solo da área experimental é apresentado na figura 4. O conteúdo de água no solo variou durante o período de estudo em função da pluviosidade local (Figura 2), corroborando o estudo realizado por Gomes (2015) nessa mesma área. A análise estatística revelou haver diferença mínima significativa das médias entre os meses estudados, porém, entre os tratamentos, não houve diferença.

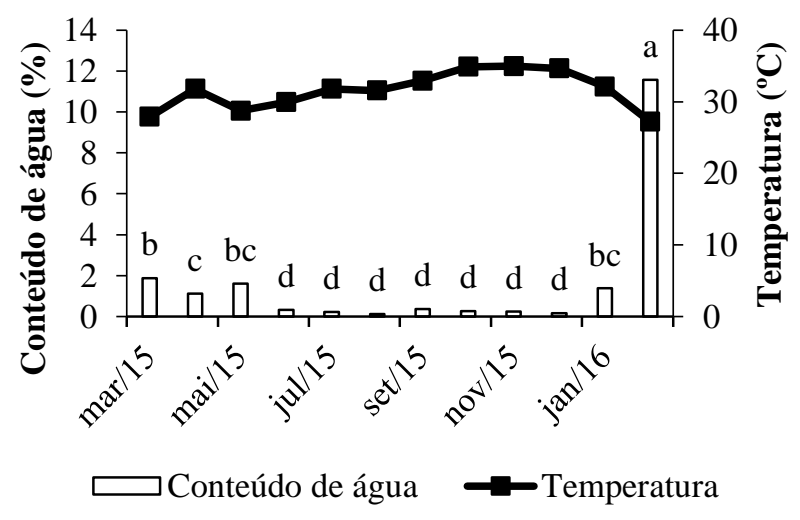

Figura 4 - Temperatura do solo e conteúdo de água do solo no período de estudo no Núcleo de Desertificação do Seridó, semiárido da Paraíba, em função da pluviosidade.

Médias seguidas de mesma letra não diferem estatisticamente entre si pelo teste de Tukey ao nível de 5\% de probabilidade.

A análise de variância do conteúdo de água no solo aponta que os valores médios do conteúdo de água nos meses de março, abril, maio/2015 e janeiro, fevereiro/2016 diferiram estatisticamente $(\mathrm{p}<0,05)$. Observa-se que os meses supracitados apresentaram o maior valor de conteúdo de água no solo (aproximadamente 2,0\%, 1,6\% 2,0\%, 2,0\% e 12,0\%, respectivamente) e também os maiores índices pluviométricos $(119,5 ; 100,0 ; 140,0 ; 280,0 \mathrm{~mm}$ e $45,0) \mathrm{e}$ as menores amplitudes térmicas, influenciadas pela interceptação da radiação solar promovida pelo material de cobertura (galhadas). A umidade do solo possui elevado grau de variabilidade no espaço e no tempo, controlada por fatores como: tempo, textura do solo, vegetação e topografia (SANTOS et al., 2011).

Nos meses de coleta, de junho a dezembro/2015, os valores médios do conteúdo de água no solo foram baixos e não apresentaram diferenças estatísticas entre si $(p<0,05)$; foram nesses meses em que se verificaram baixos índices pluviométricos e maiores temperaturas do solo. $\mathrm{O}$ solo, além de armazenar e permitir os processos de transferência de água, solutos e gases, também armazena e transfere calor (GASPARIM et al., 2005).

Carneiro et al. (2014), ao determinarem a temperatura do solo com e sem cobertura vegetal, verificaram que o conteúdo de água do solo é de suma importância, pois a presença de água afeta o fluxo de calor no solo, ou seja, a presença de umidade no solo modifica a amplitude de temperatura ao nível de superfície por ocasião da evaporação.

O maior valor do conteúdo de água foi observado no mês de fevereiro/2016, em decorrência de precipitação pluviométrica nos dias que antecederam a coleta de dados. Santos et al. (2009) afirmam que, quando ocorrem maiores insolações, constata-se menor nebulosidade, e, como consequência, tem-se uma relação inversa entre a umidade e a radiação solar global, o que também pode ser verificado pela variabilidade temporal das condições meteorológicas.

No Núcleo de Desertificação do Seridó, a temperatura média mensal mantém-se acima de $20^{\circ} \mathrm{C}$, o que é uma característica das zonas quentes, e os valores de conteúdo de água no solo são bastante baixos. As temperaturas mais elevadas ocorrem nos meses de outubro a janeiro, e as mais baixas, nos meses de maio a agosto (EMBRAPA, 2002), corroborando os resultados deste estudo. 
É importante destacar que a presença das galhadas mantem a temperatura do solo mais estável e reduz as perdas de água, devido a sua cobertura proteger a superfície do impacto direto da chuva e dos raios solares. Portanto, o solo descoberto está mais suscetível à incidência dos raios solares e perda de umidade por evaporação que, segundo a Embrapa (2002), os valores de evaporação no Núcleo de Desertificação do Seridó são bastante elevados, aproximadamente 5 vezes ou mais que os de precipitação.Lopes et al. (2011), ao estudarem a espacialização da umidade do solo por meio da temperatura da superfície e índice de vegetação, puderam verificar que a umidade do solo varia em função da cobertura vegetal, uma vez que o conteúdo de água do solo variou de 0 (zero), para as áreas desertificadas e com queimadas, até 0,57 , para áreas com vegetação.

Percebe-se, portanto, que a utilização da técnica de transposição de galhadas, nas áreas em processo de desertificação no semiárido brasileiro, torna-se uma iniciativa imprescindível, pois a técnica protegerá o solo da incidência direta de raios solares, diminuindo a perda de água por evaporação, facilitando a atividade microbiana e evitando o impacto das gotas de chuva diretamente sobre a superfície do solo.

\section{CONCLUSÕES}

Nas condições de presença de galhadas, a temperatura do solo apresentou menores amplitudes ao longo do período experimental, em relação ao solo descoberto. A temperatura no perfil do solo variou em função da profundidade e do conteúdo de água no solo.

\section{REFERÊNCIAS BIBLIOGRÁFICAS}

BELAN, L.L.; XAVIER, T.M.T.; TORRES, H.; TOLEDO, J.V.; PEZZOPANE, J.E.M. Dinâmica entre temperaturas do ar e do solo sob duas condições de cobertura. Revista Acadêmica de Ciências Agrárias e Ambientais, v. 11, n. 1, p. 147-154, 2013.

CARNEIRO, R.G.; MOURA, M.A.L.; SILVA, V.P.R.; SILVA JÚNIOR, R.S.; ANDRADE, A.M.D.; SANTOS, A.B. Estudo da temperatura do solo em dois biomas florestais nos períodos, chuvoso e seco. Revista Brasileira de Geografia Física, Recife, v. 6, p. 10091022, 2013.

CARNEIRO, R.G.; MOURA, M. A. L.; SILVA, V.P.R.; SILVA JUNIOR, R.S.; ANDRADE, A.M.D.; SANTOS, A.B. Variabilidade da temperatura do solo em função da liteira em fragmento remanescente de mata atlântica. Revista Brasileira de Engenharia Agrícola e Ambiental, v. 18, n.1, p. 99-108, 2014.

EMBRAPA. Caracterização dos recursos naturais de uma área piloto do núcleo de desertificação do Seridó,
Estados do Rio Grande do Norte e da Paraíba. Rio de Janeiro: Embrapa Solos, 2002. 53 p.

EMBRAPA. Empresa Brasileira de Pesquisa Agropecuária. Sistema Brasileiro de Classificação de Solos. Rio de Janeiro: EMBRAPA/CNPS, 2013. 353 p.

FIGUEIREDO, V.S. Perspectivas de recuperação para áreas em processo de desertificação no semiárido da Paraíba - Brasil. Scripta Nova.Revista Electrónica de Geografía y CienciasSociales, v. 17, n. 453, 2013. Disponível em: <http://www.ub.es/geocrit/sn/sn453.htm>. Acessoem: 15 out. 2014.

GASPARIM, E.; RICIERI, R.P.; SILVA, S.L.S; DALLACORT, R.; GNOATTO, E. Temperatura no perfil do solo utilizando duas densidades de cobertura e solo nu. Acta ScientiarumAgronomy,v. 27, n. 1, p. 107-115, 2005.

GOMES, R.V. Aplicação da técnica “Bocaj” e condições microclimáticas em área degradada no Seridó da Paraíba. 2015. 39 f. Trabalho de Conclusão de Curso (Graduação em Engenharia Florestal) Universidade Federal de Campina Grande, Patos, 2015.

KOPPEN, W. Climatología: conunstudio de los climas de latierra. México. Buenos Aires, Fundo de Cultura Econômica, 1948. 31p. Trad: Côrrea, A.C.B. Sistema Geográfico dos Climas. UFPE, Recife-PE, 1996. 13 p.

LOPES. H.L.; ACCIOLY, L. J.O.; SILVA, F.H.B.B.; SOBRAL, M.C.M.; ARAUJO FILHO, J.C.; CANDEIAS, A.L.B. Espacialização da umidade do solo por meio da temperatura da superfície e índice de vegetação. Revista Brasileira de Engenharia Agrícola e Ambiental,v. 15, p. 973-980, 2011.

MOURA, M.A.L.; QUERINO, C.A.S. Variação sazonal do fluxo de calor no solo dentro de um manguezal tropical. Revista Brasileira de Engenharia Agrícola e Ambiental, Campina Grande, v. 14, p. 296-302, 2010.

PEREZ-MARIN, A.M.; CAVALCANTE, A.M.B.; MEDEIROS, S.S.; TINÔCO, L.B.M.; SALCEDO, I.H.Núcleos de desertificação no semiárido brasileiro: ocorrência natural ou antrópica?.Parcerias Estratégicas, v. 17, n. 34, p. 87-106, 2012.

REIS, A.; BECHARA, F.C.; TRES, D.R. Nucleation in tropical ecological restoration. ScientiaAgricola, v. 67, n.2, p. 244-250, 2010.

REIS, A.; BECHARA, F.C.; TRES, D.R.; TRENTIN, B.E. Nucleação: concepção biocêntrica para a restauração ecológica. Ciência Florestal, v. 24, n. 2, p. 509-519, 2014.

SANTOS, D.M.; ARAUJO, S.M.; ARAUJO, K.D.; ROSA, P.R.O.; PAZERA JÚNIOR, E. Variabilidade temporal da radiação solar e insolação no município de Araguaína-TO. Revista Raega, n. 18, p. 35-40, 2009. 
SANTOS, T.E.M.; MONTENEGRO, A.A.A.; SILVA, D.D. Umidade do solo no semiárido pernambucano usando-se reflectometria no domínio do tempo (TDR). Revista Brasileira de Agrícola e Ambiental, Campina Grande, v. 7, p. 670-679, 2011.

SAS. SAS/STAT 9.3User'sGuide. Cary, NC: SAS Institute Inc. 2011, 8621 p.

SILVEIRA, L.P.; SOUTO, J.S.; DAMASCENO, M.M.; MUCIDA, D.P.; PEREIRA, I.M. Poleiros artificiais e enleiramento de galhada na restauração de área degradada no semiárido da Paraíba, Brasil. Nativa, v. 3, n. 3, p. 164$170,2015$.

RAMANA RAO, T. V.; SILVA, B. B.; MOREIRA, A. A. Características térmicas do solo em Salvador, BA. Revista Brasileira de Engenharia Agrícola e Ambiental, v.9, p.554-559, 2005. 